\title{
High Sensitivity Gas Sensor Based on IR Spectroscopy Technology and Application
}

\author{
Hengyi LI \\ Department of Electronic Information Engineering, Jincheng College of Sichuan University, 611731, Chengdu, China \\ *Corresponding author: Hengyi LI_Ｅ-mail: henrysport@163.com
}

\begin{abstract}
Due to extremely effective advantages of the quantum cascade laser spectroscopy and technology for trace gas detection, this paper presents spectroscopy scanning, the characteristics of temperature tuning, system resolution, sensitivity, and system stability with the application of the presented gas sensor. Experimental results showed that the sensor resolution was $\leq 0.01 \mathrm{~cm}^{-1}$ (equivalent to $0.06 \mathrm{~nm}$ ), and the sensor sensitivity was at the level of $194 \mathrm{ppb}$ with the application of $\mathrm{H}_{2} \mathrm{CO}$ measurement.
\end{abstract}

Keywords: Quantum cascade laser; IR spectroscopy; high sensitivity; formaldehyde

Citation: Hengyi LI, "High Sensitivity Gas Sensor Based on IR Spectroscopy Technology and Application," Photonic Sensors, DOI: 2016, 6(2): 127-131.

\section{Introduction}

Infrared laser absorption spectroscopy is an extremely effective tool for detecting trace gases. The demonstrated sensitivity of this technique is at the level of parts per billion (ppb). Presently, the usefulness of the laser spectroscopy approach is limited by the availability of convenient tunable sources in the spectroscopically important "fingerprint" region from $3 \mu \mathrm{m}$ to $20 \mu \mathrm{m}$ [1]. Recent measurements with QC-DFB (distributed feedback quantum cascade laser) lasers $[2,3]$ have demonstrated the usefulness of these devices for highly selective real-time trace gas concentration measurements based on the absorption spectroscopy with the sensitivity of several ppb [4-11].

Due to the characteristics of high output power, narrow line-width, room temperature operation, and so on, quantum cascade lasers (QCLs) have become the research focus in the fields of trace gas detection technology and instrumental integration system. This paper aims at the issues existing in the gas detection technology and instrument system research, and the key technologies in gas spectroscopy detection based on the QCL were carried out.

\section{Spectrum detection and gas sensor performance}

As to the experimental setup, the laser light was collimated by an off-axis parabolic gold mirror and then passed through a $20 \mathrm{~m}$ multi-pass white cell filled with the sample gas for detection. The radiation passed through a germanium Fabry-Pérot etalon with a free spectral range of $500 \mathrm{MHz}$. The longitudinal modes supported by an etalon with the length $d$ and refractive index $n$ were separated by the free spectral range (FSR), $v_{\mathrm{FSR}}=c / 2 n d$. A typical signal was detected when a 200-ns-long laser pulse at a wavelength around $8 \mu \mathrm{m}$ passed through an empty cell, which was used to find the nonlinear

Received: 22 September 2015 / Revised: 13 November 2015

(C) The Author(s) 2015. This article is published with open access at Springerlink.com

DOI: $10.1007 / \mathrm{s} 13320-015-0290-8$

Article type: Regular 
relation between the time and frequency axes.

\subsection{Spectrum scanning Range}

The spectroscopy scanning range was determined both by pulse current tuning and temperature tuning [11]. In order to express the spectrum scanning characteristics, 121 mTorr $\mathrm{H}_{2} \mathrm{CO}$ with the purity of $99.8 \%$ was introduced into the gas cell to obtain the absorption spectrum at different temperatures. Keeping the $\mathrm{H}_{2} \mathrm{CO}$ pressure and operation temperature unchanged, a series of scanning spectra were collected when tuning the operation temperature from $-15{ }^{\circ} \mathrm{C}$ to $20{ }^{\circ} \mathrm{C}$ with an increment of $5{ }^{\circ} \mathrm{C}$. The scanning spectrum is shown in Fig. 1, from which we can see that the scanning range will move to the longer wavelength with an increase in operation temperature.

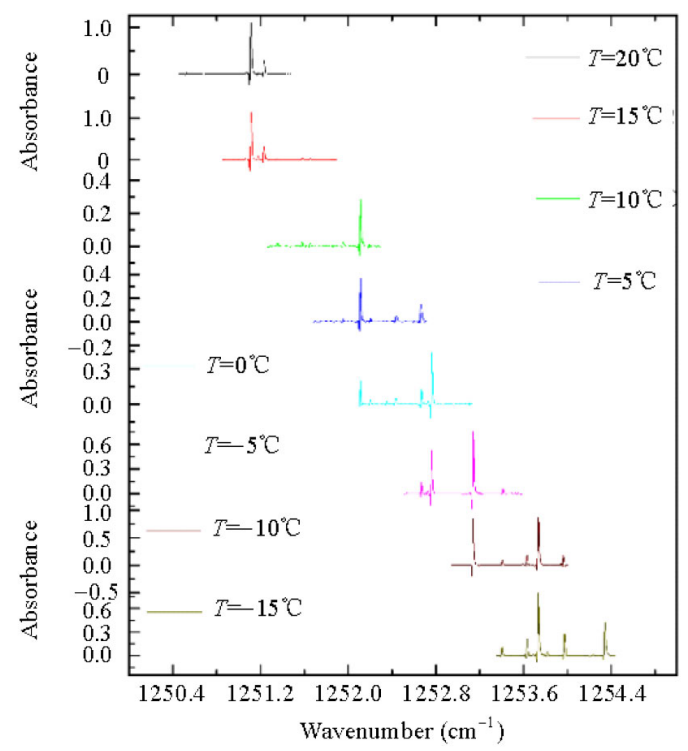

Fig. 1 Absorption line of $\mathrm{H}_{2} \mathrm{CO}$ at the sweeping temperature from $-15 \square$ to $20 \square$.

\subsection{Temperature tuning coefficient}

When the QCL operation temperature changed, the central emission wavelength would move, so it is necessary to test the temperature tuning coefficient for the detection system. Formaldehyde was used as the detection gas to get the correlation of the central emission wavelength with a function of tuning temperature, which is represented by scatter dots
“." in Fig. 2, with linear fit (represented by “-”) of the experimental data, and the calculated temperature tuning coefficient was $-0.083 \mathrm{~cm}^{-1} / \mathrm{K}$.

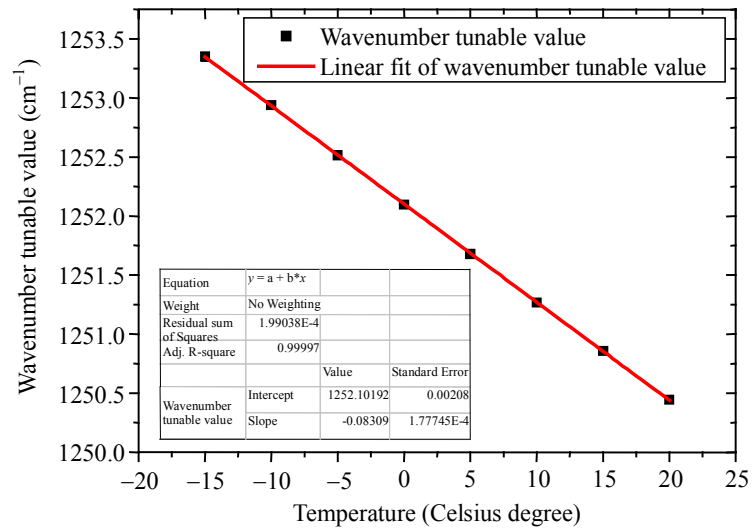

Fig. 2 Relationship between the QCL central wavenumber and temperature.

\subsection{Sensor spectrum resolution}

Spectral resolution refers to the detection system which can distinguish the minimum wavelength difference, which is based on the Rayleigh criterion, taking the absorption spectral full width at half maximum (FWHM) as the spectral resolution of the detection system. Figure 3 shows the Fourier transform spectrometer (FTIR) spectrum collected during laser operating temperature changing from $-15{ }^{\circ} \mathrm{C}$ to $+20{ }^{\circ} \mathrm{C}$, in which "—" indicates the formaldehyde absorption spectrum taken from this experimental system, and "---" indicates the spectral measurement range of $1250 \mathrm{~cm}^{-1}-$ $1255 \mathrm{~cm}^{-1}$.

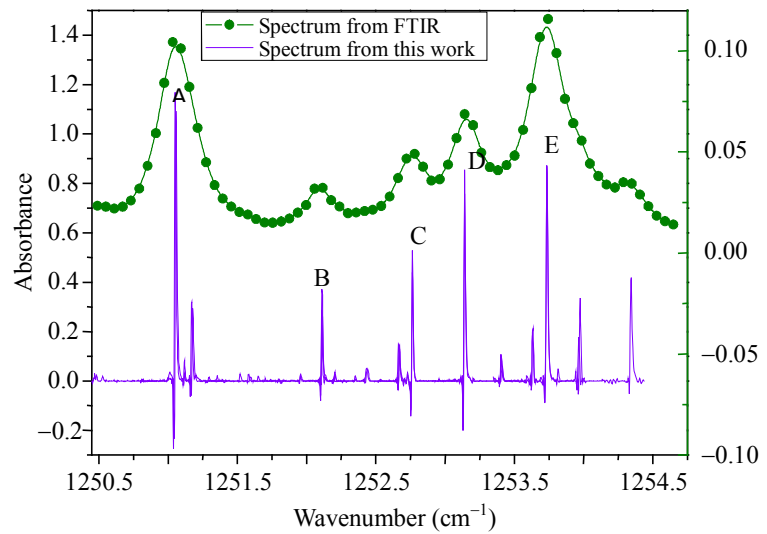

Fig. 3 Comparison of the absorption spectroscopy done in this work and FTIR. 
The calculation of formaldehyde absorption lines $\mathrm{A}, \mathrm{B}, \mathrm{C}, \mathrm{D}$, and $\mathrm{E}$ at $20{ }^{\circ} \mathrm{C}$ showed that the largest width was $0.01 \mathrm{~cm}^{-1}$, which was exactly in the most intensive areas, in which it would be more reasonable to calculate the resolution. Other results are shown in Table 1. So the resolution of this spectroscopic detection system was $\leq 0.01 \mathrm{~cm}^{-1}$ (equivalent to $0.06 \mathrm{~nm}$ ), which could also be seen from the comparison between the two results. It is obvious that the QCL detection system has a significantly higher resolution than the FTIR spectrometer.

Table 1 Linewidth of experimental formaldehyde spectrum.

\begin{tabular}{cccccc}
\hline Line & A & B & C & D & E \\
\hline FWHM $\left(\mathrm{cm}^{-1}\right)$ & 0.0100 & 0.0098 & 0.0097 & 0.0098 & 0.0096 \\
\hline
\end{tabular}

\subsection{Gas sensor stability}

The system stability renders the basic response throughout the detection time, mainly measured through the zero drift and interval drift. The zero drift refers to the absence of the measured gas and the output changes throughout the detection time of the system. The interval drift refers to the changes in the continuous output in the case of measured gas presence over the measuring time. In the absence of any absorbed gas existing, the zero drift is expressed by the output light intensity versus time shown in Fig. 4(a). In the case of $\mathrm{H}_{2} \mathrm{CO}$ gas absorption as presence, the interval drift is analyzed from the relationship of the specific absorption peak intensity varying with time, as shown in Fig. 4(b).

It can be seen from the linear fit results that the quantum cascade laser output light intensity of the standard deviation is 0.0188 , the calculated "zero drift" relative standard deviation is $0.26 \%$, and the stability of the standard deviation of gas absorption peak is 0.0007 . Thus the calculated "interval drift" expressed by relative standard deviation is $0.24 \%$. Both results illustrate a good stability of the QCL detection system.

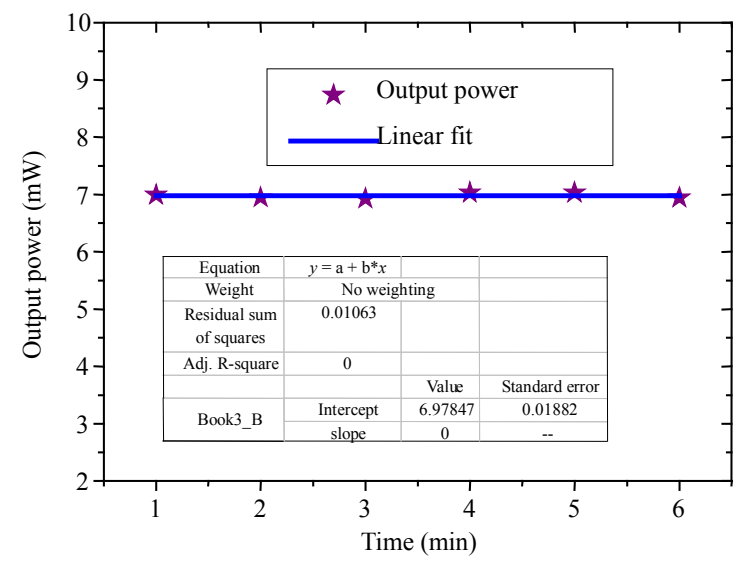

(a)

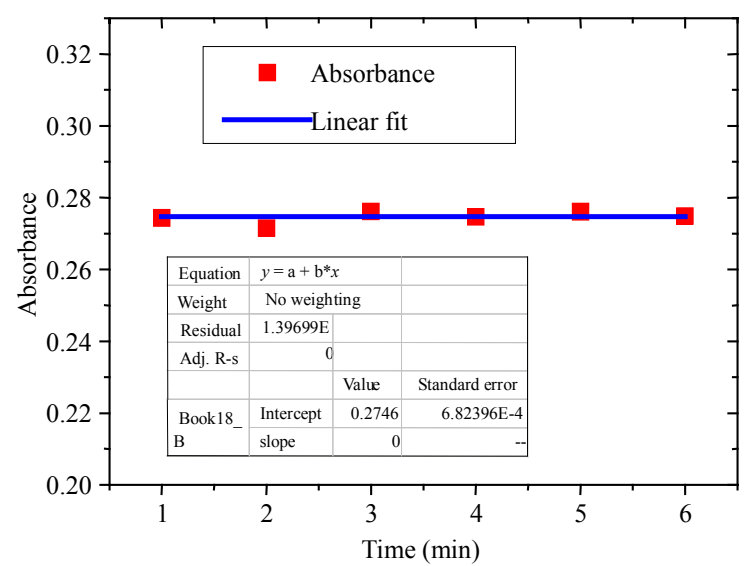

(b)

Fig. 4 System stability measured by (a) zero drift: output power of QCL varying with time and (b) interval drift: absorbance varying with time.

\subsection{Sensor sensitivity}

Selecting the appropriate line and fitting function is very important for the calculation of detection limit. Usually, we choose the Voigt function for nonlinear fitting and also the integration of spectral lines in the region, and then we obtain the integrated absorption intensity area for calculating detection sensitivity. The measured gas pressure is fixed, by varying the buffer gas pressure, so we can get different concentrations of formaldehyde.

As shown in Fig. 5(a), the absorption spectrum of $60 \mathrm{mTorr}_{2} \mathrm{CO}$ in 200 Torr $\mathrm{N}_{2}$ was measured and then fitted with the nonlinear Voigt function and non-absorption part fitted with linear function. In Fig. 5, “....." indicates original absorption lines of $\mathrm{H}_{2} \mathrm{CO}$, “—” indicates Voigt function fitting, and 
“- $\ldots$ is a linear fit to the noise signal. As seen from Fig. 5, the Voigt function fits well with the experimental results. Figure 5(b) shows fitting residuals with the deviation in the range of $0.4 \%$. After data processing and analysis, the calculated minimum detectable concentration is $187 \mathrm{ppb}$. In this system, the signal to noise ratio (SNR) is calculated to be 1549 , and the corresponding concentration is $300 \mathrm{ppm}$, then the detection sensitivity is $194 \mathrm{ppb}$ when SNR is 1 .

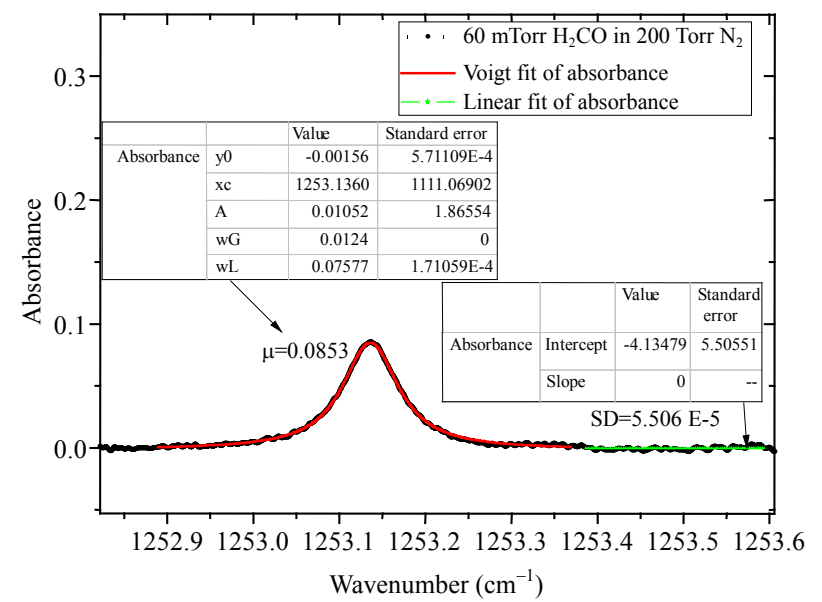

(a)

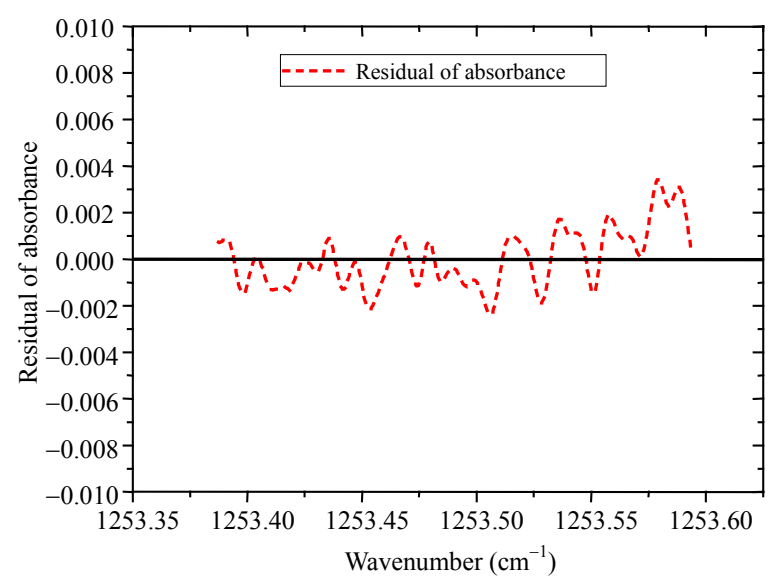

(b)

Fig. 5 Formaldehyde spectrum and the residual signal: (a) Voigt fit of 60 mTorr formaldehyde in 200 Torr $\mathrm{N}_{2}$ and (b) standard error less than $0.4 \%$ between the experimental results and Voigt function fit.

\section{Conclusions}

In this work, the gas sensor performance based on the IR spectroscopy technology was carried out. The spectrum range was $1249 \mathrm{~cm}^{-1}-1257 \mathrm{~cm}^{-1}$, the temperature tuning coefficient was $-0.083 \mathrm{~cm}^{-1} / \mathrm{K}$, the absorbance reproducibility relative standard deviation was less than $0.0051 \%$, the system resolution was less than $0.01 \mathrm{~cm}^{-1}$, and the system detects sensitivity reached $194 \mathrm{ppb}$, which was comparatively high sensitivity for detection trace gas or toxic gases.

\section{Acknowledgment}

The authors wish to thank the financial support from the National Natural Science Foundation of China (61505020) and the Fundamental Research Funds for the Central Universities (ZYGX2013J007).

Open Access This article is distributed under the terms of the Creative Commons Attribution 4.0 International License (http:// creativecommons.org/licenses/by/4.0/), which permits unrestricted use, distribution, and reproduction in any medium, provided you give appropriate credit to the original author(s) and the source, provide a link to the Creative Commons license, and indicate if changes were made.

\section{References}

[1] J. Faist, F. Capasso, D. L. Sivco, C. Sirtori, A. L. Hutchinson, and A. Y. Cho, "Quantum cascade laser," Science, 1994, 264(158): 553-556.

[2] L. Wang and T. R. Sharples, "Intrapulse quantum cascade laser spectroscopy: pressure induced line broadening and shifting in the $v 6$ band of formaldehyde," Applied Physics B, 2012, 108(2): 427-435.

[3] T. Steck, N. Glatthor, T. von Clarmann, H. Fischer, J. M. Flaud, and B. Funke, "Retrieval of global upper tropospheric and stratospheric formaldehyde $\left(\mathrm{H}_{2} \mathrm{CO}\right)$ distributions from high-resolution MIPAS-envisat spectra," Atmospheric Chemistry \& Physics, 2008, 8(3): 463-470.

[4] X. Chen, L. Cheng, D. Guo, Y. Kostov, and F. S. Choa, "Quantum cascade laser based standoff photoacoustic chemical detection," Optics Express, 2011, 9(21): 20251-20257.

[5] L. Wang and S. Thomas-Reoben, "Monitoring hydrogen sulfide using a quantum cascade laser based trace gas sensing system," Chinese Physics Letters, 2011, 28(6): 1183-1187.

[6]A. Wisthaler, E. C. Apel, J. Bossmeyer, A. Hansel, W. Junkermann, and R. Koppmann, "Technical note: 
intercomparison of formaldehyde measurements at the atmosphere simulation chamber SAPHIR," Atmospheric Chemistry \& Physics Discussions, 2008, 7(6): 15619-15650.

[7] K. Chance and J. Orphal, "Revised ultraviolet absorption cross sections of $\mathrm{H}_{2} \mathrm{CO}$ for the HITRAN database," Journal of Quantitative Spectroscopy \& Radiative Transfer, 2011, 112(9): 1509-1510.

[8] I. R. Burling, R. J. Yolelson, S. K. Akagi, S. P. Urbanski, C. E. Wold, D. W. T. Griffith, et al., "Airborne and ground-based measurements of the trace gases and particles emitted by prescribed fires in the United States," Atmospheric Chemistry \& Physics, 2011, 11(23): 12197-12216.
[9] C. L. Heald, A. H. Goldstein, J. D. Allan, and A. C. Aiken, "Total observed organic carbon (TOOC) in the atmosphere: a synthesis of North American observations," Atmospheric Chemistry \& Physics, 2008, 8(7): 2007-2025.

[10] L. Wang, "Formaldehyde and methane spectroscopy measurements based on mid-IR quantum cascade laser system," Journal of Infrared and Millimeter Waves, 2014, 33(6): 591 -597.

[11] G. Duxbury, N. Langford, M. T. McCulloch, and W. Stephen, "Quantum cascade semiconductor infrared and far-infrared lasers: from trace gas sensing to non-linear optics," Chemical Society Reviews, 2005, 34(11): 921-934. 